\title{
Black Men Who Have Sex with Men and Lifetime HIV Testing: Characterizing the Reasons and Consequences of Having Never Tested for HIV
}

\author{
Derrick D. Matthews ${ }^{1}$ (1) - Jordan M. Sang ${ }^{2,3} \cdot$ Cristian J. Chandler $^{4} \cdot$ Leigh A. Bukowski $^{2,3} \cdot$ M. Reuel Friedman ${ }^{2,5}$. \\ Lisa A. Eaton ${ }^{6} \cdot$ Ron D. Stall ${ }^{2,3}$
}

Published online: 14 May 2019

\begin{abstract}
HIV testing remains a critical point of entry to HIV treatment services and now biomedical prevention as well. Yet despite the high HIV prevalence among Black men who have sex with men (MSM), insufficient attention has been given to factors associated with those Black MSM in the United States who have never received an HIV test in their lifetime. Promoting Our Worth, Equality, \& Resilience (POWER) is a cross-sectional observational study that recruited Black MSM at Black Pride events across six cities in the United States from 2014 to 2017. Participants completed an anonymous questionnaire and were offered free, confidential HIV testing. Of the 4174 Black MSM without a prior HIV diagnosis, 404 (9.68\%) had never tested for HIV (mean age $=31.03$ years). Lower education and greater internalized homophobia were associated with never having tested for HIV. Higher age $(\mathrm{AOR}=1.05,95 \%, 1.02-1.07)$ and assumption of HIV-positivity (AOR $=3.24,95 \%$ CI 1.53-6.84) were both associated with increased odds of an HIV-positive test result $(n=119 ; 36 \%)$. To compare, HIV prevalence among Black MSM who had received at least one HIV test before study participation was $23 \%$. While a minority of Black MSM had never received an HIV test, this group had a significantly higher likelihood of HIV infection. Alternative HIV testing strategies are needed to facilitate HIV testing initiation among Black MSM for whom conventional HIV testing modalities are insufficient.
\end{abstract}

Keywords HIV/AIDS $\cdot$ Black MSM $\cdot$ HIV testing $\cdot$ Health disparities

\section{Introduction}

Black men who have sex with men (MSM) have a 1 in 2 lifetime risk of becoming HIV-positive (Hess et al. 2017).

Derrick D. Matthews

derrick.matthews@unc.edu

1 Department of Health Behavior, The University of North Carolina Gillings School of Global Public Health, Chapel Hill, NC, USA

2 Center for LGBT Health Research, University of Pittsburgh Graduate School of Public Health, Pittsburgh, PA, USA

3 Department of Behavioral and Community Health Sciences, University of Pittsburgh Graduate School of Public Health, Pittsburgh, PA, USA

4 Department of Epidemiology, Emory University Rollins School of Public Health, Druid Hills, GA, USA

5 Department of Infectious Diseases and Microbiology, University of Pittsburgh Graduate School of Public Health, Pittsburgh, PA, USA

6 Department of Human Development and Family Studies, University of Connecticut, Mansfield, CT, USA
Crucial to any strategy to address this profound disparity is the timely identification of HIV infection through accessible HIV testing services for Black MSM.

Though rates of HIV testing have increased over time, there remains a notable minority of MSM who have never been tested for HIV, especially those under 25 (Hammack et al. 2018). Research into the barriers for regular HIV testing rarely separates those who have never received an HIV test in their lifetime from those who have not received one in the past 6 or 12 months. Studies that do have found associations between no lifetime history of HIV testing and condomless anal intercourse, a lack of resources (e.g., money, health insurance), and not knowing where to go to receive an HIV test (Margolis et al. 2012; MacKellar et al. 2011; Clark et al. 2018). Notably MSM also report a low perception of risk and fear of being HIV-positive as reasons for never having received an HIV test, with the latter especially true among Black MSM (MacKellar et al. 2011). Of additional concern, Black MSM are estimated to make up nearly $30 \%$ of those MSM with no history of HIV testing (Clark et al. 2018).

For this study, we address the challenge of enrolling those with no history of HIV testing: that they are among the least 
likely to engage with HIV research. In the event someone declined HIV testing, we allowed an alternative method of participation, ensuring their reticence towards HIV testing would not preclude their ability to contribute to research. We use this exploratory study to characterize factors associated with having never received an HIV test and assess the HIV prevalence among this group of understudied and underserved group of Black MSM.

\section{Methods}

\section{Study Design and Population}

Data come from Promoting Our Worth, Equality, and Resilience (POWER), a serial cross-sectional, communityengaged study of Black MSM and Black transgender women. Participants were recruited from lesbian, gay, bisexual, and transgender (LGBT) Black Pride events organized by and for Black LGBT communities. Individuals were eligible to participate if they (1) were assigned male sex at birth, (2) reported having either anal or oral sex with male sexual partner in their lifetime, and (3) were 18 years of age or older. POWER's recruitment strategy, including the process used to identify and remove repeat respondents, has been detailed elsewhere (Turner et al. 2003; Eaton et al. 2018). This study of HIV testing behavior only includes those who (1) identified as "Black or African American", (2) identified as male, (3) and reported an HIV status other than HIV-positive.

Upon completion of the questionnaire, POWER offered every participant confidential HIV testing performed onsite by local community-based organizations (CBOs). CBOs utilized their own rapid HIV-testing protocol, using either the Oraquick (OraSure Technologies, Inc., Bethlehem, PA), Clearview STAT-PAK (Alere Inc., Waltham, MA), or INSTI (bioLythical Laboratories, Richmond, BC) HIV test. Confirmatory testing of preliminary positive results occurred offsite later using existing protocols; these confirmatory results were used exclusively for diagnosis and care linkage and were not provided to POWER. Participants electing confidential HIV testing received their test result and \$20. Only in the event a participant declined confidential HIV testing, POWER staff offered a modified form of "anonymous" HIV testing of oral fluid via OraQuick in which participants would only receive the $\$ 20$ but not their test result. Participants declining either form of HIV testing were paid $\$ 10$ for completion of the survey. HIV test results were anonymously linked to survey files via a unique subject ID.

\section{Measures}

Having ever received an HIV test was assessed with the following yes/no item: "Have you ever been tested for HIV?"
Guess of HIV status was assessed among participants who indicated they had never tested for HIV with the following item: "If you had to guess, what do you think your HIV status is?" with response options "HIV-positive" and "HIV-negative."

Most important reason for not having tested for HIV was assessed by asking participants to select one of the following options as the important reason for never having tested for HIV: (1) "You think you are at low risk for HIV infection;" (2) "You were afraid of finding out you had HIV;" (3) "You didn't have time;" (4) "You were afraid of others finding out;" (5) "You were afraid others would judge you;" (6) "You didn't trust the test to be accurate;" (7) "Some other reason;" and (8) "No particular reason."

HIV status was operationalized using the result of the HIV test conducted as part of study participation.

Election to receive HIV test result assessed whether participants tested for HIV with the local CBO and received their HIV test results or chose to provide oral fluid for POWER staff to process with OraQuick and did not receive HIV test results. Participants could also decline all forms of HIV testing.

Sexual orientation identity was assessed with the following item: "Which of the following do you identify as?" with the following four response options: (1) "gay/same gender loving," (2) "heterosexual or "straight," (3) "bisexual," and (4) "other."

Presence of health insurance coverage was assessed with the following yes/no item: "Do you currently have health insurance or health care coverage?"

Depressive symptomatology was assessed using the Center for Epidemiologic Studies Depression 10 (CES-D 10; Cronbach's $\alpha=0.75$ ). As recommended, this scale was dichotomized so that individuals with scores of 10 or greater were considered to have sufficient depressive symptomatology consistent with major or clinical depression (Zhang et al. 2012).

Internalized homophobia, the degree to which participants reported negative views of their sexual orientation, was assessed with the nine-item Internalized Homophobia Scale (Cronbach's $\alpha=0.92$; e.g., "I wished I weren't attracted to men.") (Herek et al. 1998).

Demographic covariates including age (in years), education ( $<$ high school or GED, high school/GED, some college or technical school, college graduate), city, and year of data collection were also assessed.

\section{Analysis}

Bivariate comparisons were made between those who had ever tested for HIV in their lifetime to those who had never tested for HIV; among the subsample of those with no HIV testing history, we further examined differences by guess of 
HIV status, decision to engage in HIV testing, and HIV test result. Multivariable logistic regression was used to make these comparisons while simultaneously adjusting for all study variables. We also report the reasons Black MSM indicated for never having tested for HIV. All analysis were conducted in SAS version 9.4, (SAS Institute, Inc., Cary, NC), with all significance set at alpha $=0.05$.

\section{Results}

\section{Sample Characteristics}

The inclusion criteria for this study resulted in an analytic sample of 4174 Black MSM. A more detailed distribution of the analytic sample can be seen in Fig. 1. Table 1 presents sample characteristics. A majority $(n=3770,90.32 \%)$ reporting having ever tested for HIV. Compared to those with a history of at least one HIV test, "never testers" were less likely to have completed education past high school $\left(\chi^{2}=\right.$ $120.90, p<.05)$, and were also less likely to have a form of health insurance (AOR $=0.67,95 \%$ CI $0.52-0.87$ ). Both internalized homophobia (AOR $=1.36,95 \%$ CI $1.21-1.52)$ and depressive symptomatology ( $\mathrm{AOR}=1.45,95 \% \mathrm{CI} 1.13-1.85$ ) were associated with a greater likelihood of having never tested for HIV.

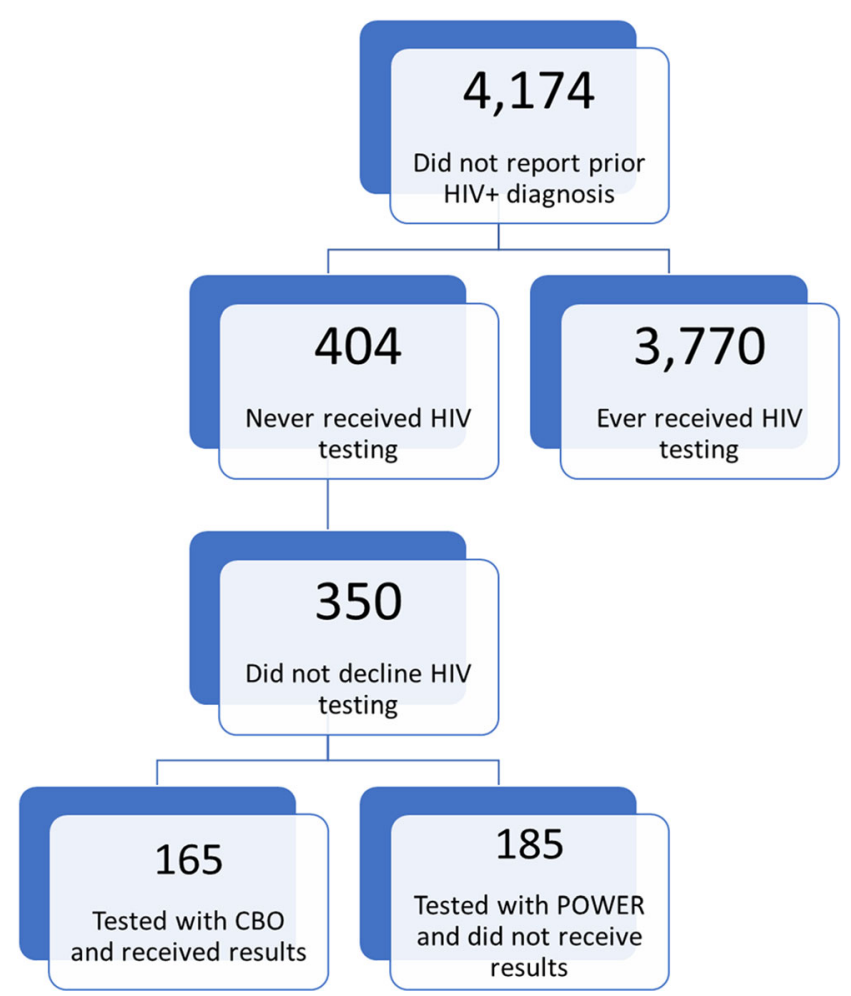

Fig. 1 Distribution of POWER sample, six US cities from 2014 to 2017: Black MSM without a prior HIV-positive diagnosis
Among the 404 Black MSM who never tested for HIV, 396 (98.02\%) reported their guess of their HIV status. Age was positively associated with the belief they were HIV positive (AOR $=1.06,95 \%$ CI 1.04-1.09). Figure 2 displays the list of the most important reasons Black MSM endorsed for having never tested for HIV. The most common reason among those who guessed they were HIV-negative was a belief that they were at low risk for HIV infection, while the most common reason for those who guessed they were HIV-positive was being afraid of finding out they were HIV-positive. Reasons for never having received an HIV test did not differ whether participants tested with POWER or a local CBO (data not shown).

A minority $(n=54)$ of the Black MSM who had never tested for HIV declined to select any HIV testing option; the remaining ( $n=350,86.64 \%)$ accessed an HIV testing option provided by POWER or a local CBO associated with the study. The option to provide POWER with an anonymous oral sample (and not receive HIV test result) $(n=185 ; 45.79 \%)$ was more popular than choosing to receive HIV testing through a local CBO (and receive HIV test result) ( $n=165$; $40.84 \%$ ). There were no significant associations with the decision to receive HIV testing at time of study participation.

Among Black MSM who tested for HIV for the first time through their participation in the study, age was associated with a greater likelihood of being HIV-positive (AOR = $1.05,95 \%, 1.02-1.07)$, as was guessing one was HIVpositive (AOR $=3.24,95 \% \mathrm{CI}: 1.53-6.84)$. For those Black MSM who did receive their first HIV test through participation in the study, about one-third $(n=119 ; 35.74 \%)$ had an HIV-positive test result. To contrast, the HIV prevalence among those who ever tested for HIV test prior to study participation was $23.42 \%,\left(\chi^{2}=22.87, p<.01\right)$.

\section{Discussion}

Results from this study provide useful information into factors associated with never having received an HIV test. Though a minority of Black MSM (9.68\%) had no lifetime history of HIV testing, our findings expand the literature that has recognized the importance of those living with HIV who do not yet know their status as having an oversized effect in sustaining new HIV infections (Skarbinski et al. 2015). Nearly a third of Black MSM who had never tested prior to this study were determined to be HIV-positive, though we draw attention that a 23\% HIV prevalence among Black MSM with a history of HIV testing remains unacceptably high.

We found few characteristics that may facilitate identification in a clinical setting, though the average age of these men was 31-years old, suggesting multiple missed opportunities over many years. HIV stigma remains a powerful and prevalent deterrent to HIV testing (Mahajan et al. 2008); men in our 
Table 1 Characteristics of self-identified HIV-negative and HIV-unknown Black MSM by HIV testing history, six US cities from 2014 to 2017 ( $n=$ 4174)

Never had an HIV test Had at least one HIV test (ref.) Odds ratio (95\% CI) Adjusted odds ratio (95\% CI)

\begin{tabular}{|c|c|c|c|c|}
\hline Total, No. $(\%)$ & $404(9.68)$ & $3770(90.32)$ & & \\
\hline Age, mean (SE) & $31.03(0.59)$ & $30.32(0.15)$ & $1.00(0.98-1.02)$ & $1.00(0.99-1.02)$ \\
\hline \multicolumn{5}{|l|}{ Education, No. (\%) } \\
\hline$<$ High school/GED & $69(17.12)$ & $225(6.03)$ & $5.24(3.69-7.42)$ & $3.13(2.14-4.58)$ \\
\hline High school/GED & $139(34.49)$ & $744(19.94)$ & $3.19(2.40-4.25)$ & $2.55(1.87-3.48)$ \\
\hline Some college or technical school & $112(27.79)$ & $1345(36.05)$ & $1.42(1.06-1.91)$ & $1.31(0.96-1.78)$ \\
\hline College graduate & $83(20.60)$ & $1417(37.98)$ & 1.00 & 1.00 \\
\hline \multicolumn{5}{|l|}{ Sexual orientation identity } \\
\hline Bisexual & $112(27.79)$ & $686(18.20)$ & $1.79(1.42-2.27)$ & $1.25(0.96-1.61)$ \\
\hline Heterosexual & $12(2.98)$ & $48(1.27)$ & $2.79(1.46-5.33)$ & $1.23(0.60-2.50)$ \\
\hline Other & $10(2.48)$ & $60(1.59)$ & $1.82(0.92-3.60)$ & $1.31(0.62-2.76)$ \\
\hline Gay or same-gender loving & $269(66.75)$ & $2976(78.94)$ & 1.00 & 1.00 \\
\hline Health insurance access, No. (\%) & $296(73.45)$ & $3189(84.64)$ & $0.50(0.40-0.64)$ & $0.67(0.52-0.87)$ \\
\hline Internalized homophobia, ${ }^{\mathrm{a}}$ mean (SE) & $1.85(0.05)$ & $1.40(0.02)$ & $1.55(1.40-1.72)$ & $1.36(1.21-1.52)$ \\
\hline Depressive symptomatology, No. (\%) & $125(31.33)$ & $666(17.76)$ & $2.11(1.68-2.65)$ & $1.45(1.13-1.85)$ \\
\hline
\end{tabular}

Significant results are italicized (at least $p<.05$ )

Note: Multivariable model additionally adjusts for city and year of data collection

${ }^{\mathrm{a}}$ Range $=0-4$, with higher values indicative of greater internalized homophobia

study endorsed being "afraid others would judge you" or "afraid of others finding out" as reasons for not testing (Earnshaw and Chaudoir 2009). Integrating HIV testing into routine care could drastically reduce the number of persons who have not been HIV tested nationally and bypass trying to identify these men specifically. By creating a positive and stigma-free initial encounter, we can increase the likelihood not only that Black MSM will return for HIV test results, but that we can successfully offer additional services such as testing for other sexually transmitted infections or pre-exposure prophylaxis (PrEP) initiation.

Our sampling strategy recruited a diversity of Black MSM though results are not generalizable to all Black MSM. Those who tested with POWER and local CBOs did not differ in

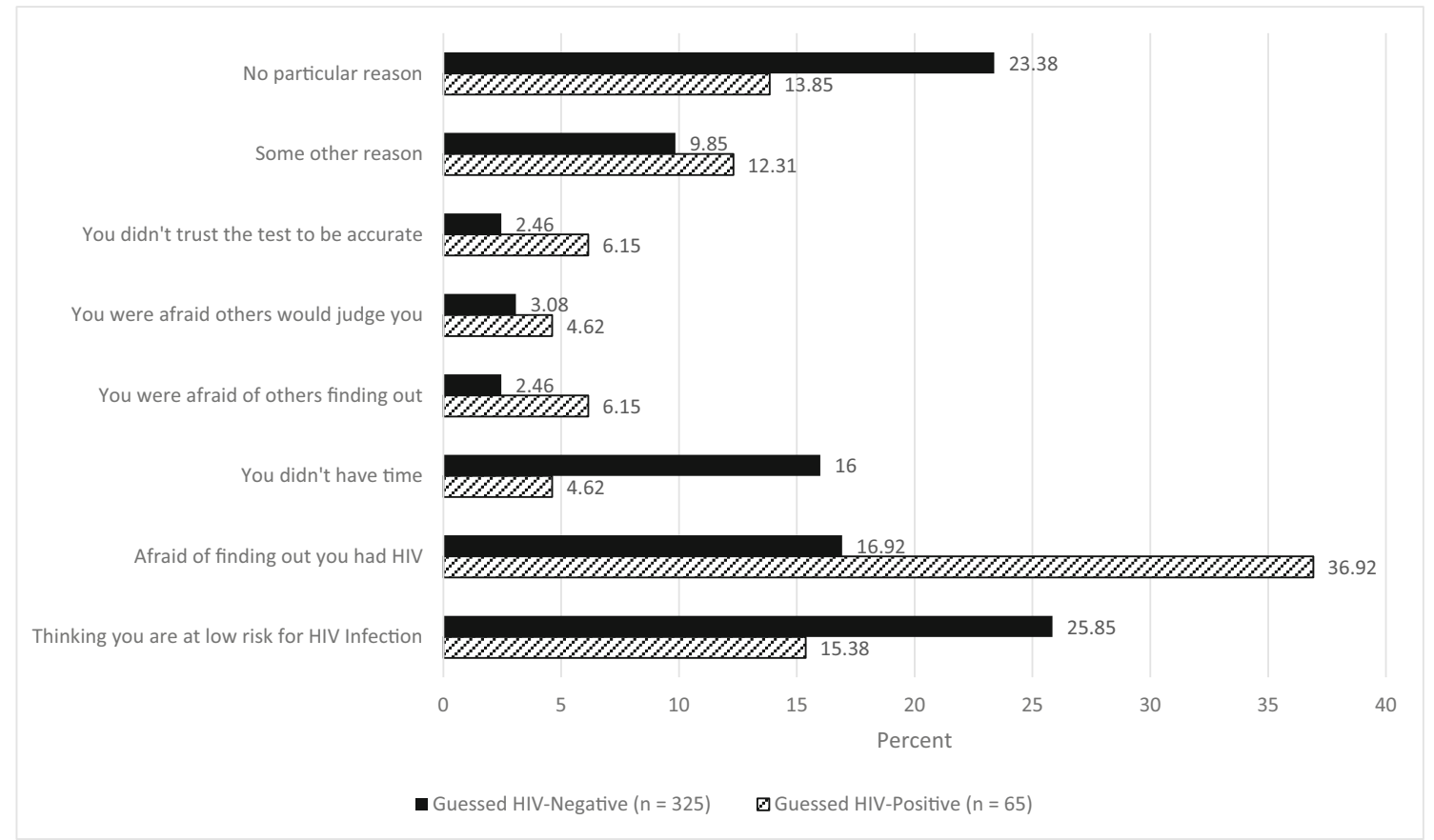

Fig. 2 Most important reason Black MSM reported for never testing for HIV, six US cities from 2014 to $2017(N=390)$ 
their reported reasons for never HIV testing; though because participants were forced to choose one primary reason, we may not be detecting differences if they exist. We rely on self-report data; though arguably, it may have resulted an underestimation of the number of Black MSM who have never received an HIV test. Finally, POWER only assessed details of sexual behavior in the past year, so we cannot determine if a sizeable proportion of men who have never received an HIV test consistently engaged in sex with condoms or exclusively had oral sex, though the heightened HIV prevalence among this group would suggest this is unlikely.

Despite the limitations of this cross-sectional design, our ability to recruit thousands of Black MSM from Black Pride events resulted in the ability to identify those who have never tested for HIV. Approximately a quarter of those with no HIV testing history endorsed "no particular reason" or "some other reason," pointing to qualitative research as a crucial next step to understand the context that results in a lack of HIV testing for Black MSM. There are factors related to low health care utilization for Black men broadly that we did not explore such as medical mistrust (Hammond et al. 2010). That those who believed they were HIV-positive were more likely to have an HIV-positive test result is a notable finding. Not only may suspicion of HIV infection be a barrier to testing, but conversely so too is low risk perception-most men who tested HIV-positive thought they were HIV-negative. Beyond messaging, interventions need to address the underlying stigma and structural barriers that keep some from HIV testing despite the treatable and chronic nature of HIV infection.

Despite many remaining challenges, our study was able to identify hundreds of Black MSM with no history of HIV testing, and the CBOs we partnered with were able to provide HIV test results to 165 of these men. Through repeated presence in spaces where Black MSM socialize, an enhanced community-engaged form of venue-based testing has potential to reach those for whom public health services have remained difficult to access.

Acknowledgements We express our appreciation to the thousands of participants who donated their time in our research, and to the Center for Black Equity for welcoming our research team into the community of Black Prides across the country. We extend a special thanks to the dozens of service organizations who provided HIV testing services for our study participants.

Compliance with Ethical Standards This study was approved by the University of Pittsburgh's IRB. All procedures performed in studies involving human participants were in accordance with the ethical standards of the institutional and/or national research committee and with the 1964 Helsinki declaration and its later amendments or comparable ethical standards. Informed consent was obtained from all individual participants included in the study. This research was supported by the National Institute of Nursing Research (grant R01NR013865), the National Institute of Allergy and Infectious Diseases (grant R21AI120777), and the National Institute of Mental Health (grant T32MH094174).
Conflict of Interest The authors declare that they have no conflict of interest.

\section{References}

Clark, H. A., Oraka, E., DiNenno, E. A., Wesolowski, L. G., Chavez, P. R., Pitasi, M. A., et al. (2018). Men who have sex with men (MSM) who have not previously tested for HIV: Results from the MSM testing initiative, United States (2012-2015). AIDS and Behavior, 1-7.

Earnshaw, V. A., \& Chaudoir, S. R. (2009). From conceptualizing to measuring HIV stigma: A review of HIV stigma mechanism measures. AIDS and Behavior, 13(6), 1160-1177.

Eaton, L. A., Matthews, D. D., Bukowski, L. A., Friedman, M. R., Chandler, C. J., Whitfield, D. L., et al. (2018). Elevated HIV prevalence and correlates of PrEP use among a community sample of black men who have sex with men. Journal of Acquired Immune Deficiency Syndromes, 79(3), 339-346.

Hammack, P. L., Meyer, I. H., Krueger, E. A., Lightfoot, M., \& Frost, D. M. (2018). HIV testing and pre-exposure prophylaxis (PrEP) use, familiarity, and attitudes among gay and bisexual men in the United States: A national probability sample of three birth cohorts. PLoS One, 13(9), e0202806.

Hammond, W. P., Matthews, D., Mohottige, D., Agyemang, A., \& CorbieSmith, G. (2010). Masculinity, medical mistrust, and preventive health services delays among community-dwelling African-American men. Journal of General Internal Medicine, 25(12), 1300-1308.

Herek, G. M., Cogan, J. C., Gillis, J. R., \& Glunt, E. K. (1998). Correlates of internalized homophobia in a community sample of lesbians and gay men. Journal of the Gay and Lesbian Medical Association, 2(1), 17-25.

Hess, K. L., Hu, X., Lansky, A., Mermin, J., \& Hall, H. I. (2017). Lifetime risk of a diagnosis of HIV infection in the United States. Annals of Epidemiology, 27(4), 238-243.

MacKellar, D. A., Hou, S.-I., Whalen, C. C., Samuelsen, K., Sanchez, T., Smith, A., et al. (2011). Reasons for not HIV testing, testing intentions, and potential use of an over-the-counter rapid HIV test in an internet sample of men who have sex with men who have never tested for HIV. Sexually Transmitted Diseases, 38(5), 419-428.

Mahajan, A. P., Sayles, J. N., Patel, V. A., Remien, R. H., Ortiz, D., Szekeres, G., et al. (2008). Stigma in the HIV/AIDS epidemic: A review of the literature and recommendations for the way forward. AIDS, 22(Suppl 2), S67-S79.

Margolis, A. D., Joseph, H., Belcher, L., Hirshfield, S., Chiasson, M. A. J. A., \& Behavior (2012). 'Never testing for HIV'among men who have sex with men recruited from a sexual networking website, United States. AIDS and Behavior, 16(1), 23-29.

Skarbinski, J., Rosenberg, E., Paz-Bailey, G., Hall, H. I., Rose, C. E., Viall, A. H., et al. (2015). Human immunodeficiency virus transmission at each step of the care continuum in the United States. JAMA Internal Medicine, 175(4), 588-596.

Turner, K. R., McFarland, W., Kellogg, T. A., Wong, E., Page-Shafer, K., Louie, B., et al. (2003). Incidence and prevalence of herpes simplex virus type 2 infection in persons seeking repeat HIV counseling and testing. Sexually Transmitted Diseases, 30(4), 331-334.

Zhang, W., O'Brien, N., Forrest, J. I., Salters, K. A., Patterson, T. L., Montaner, J. S., et al. (2012). Validating a shortened depression scale (10 item CES-D) among HIV-positive people in British Columbia, Canada. PLoS One, 7(7), e40793.

Publisher's Note Springer Nature remains neutral with regard to jurisdictional claims in published maps and institutional affiliations. 